\title{
The microRNA miR-516a-3p regulates the Wnt pathway by targeting extracellular sulfatase 1 in human scirrhous gastric cancers: Anti-metastatic therapy via miRNA-based medicine
}

\author{
Authors: \\ Yoshifumi Takei $^{1,2 *}$, Akiko Suzuki $^{1,2}$, Keichiro Mihara ${ }^{3}$, Kazuyoshi Yanagihara $^{4}$
}

\begin{abstract}
Affiliations:
${ }^{1}$ Department of Medicinal Biochemistry, School of Pharmacy, Aichi Gakuin University, 1-100 Kusumoto-cho, Chikusa-ku, Nagoya 464-8650, Japan

${ }^{2}$ Division of Disease Models, Center for Neurological Diseases and Cancer, Nagoya University Graduate School of Medicine, Showa-ku, Nagoya 466-8550, Japan

${ }^{3}$ Department of Hematology and Oncology, Research Institute for Radiation Biology and Medicine, Hiroshima University, 1-2-3 Kasumi, Minami-ku, Hiroshima 734-8553, Japan

4 Division of Biomarker Discovery, Exploratory Oncology and Clinical Trial Center, National Cancer Center Hospital East, 6-5-1 Kashiwanoha, Kashiwa, Chiba, 277-8577, Japan
\end{abstract}

Journal category: Original paper

Key words: microRNA (miRNA), scirrhous gastric cancer, peritoneal dissemination, Wnt pathway, $\beta$-catenin, anti-metastatic therapy

\section{Corresponding author:}

Yoshifumi Takei, Ph.D.

Department of Medicinal Biochemistry, School of Pharmacy, Aichi Gakuin University, 1-100 Kusumoto-cho, Chikusa-ku, Nagoya 464-8650, Japan.

Phone: 81-52-751-2561. Fax: 81-52-757-6799.

E-mail: takei@dpc.agu.ac.jp 
Medical Research Archives, Vol. 5, Issue 7, July 2017

The microRNA miR-516a-3p regulates the Wnt pathway by targeting extracellular sulfatase 1 in human scirrhous gastric cancers: Anti-metastatic therapy via miRNA-based medicine

\begin{abstract}
The mechanism and function of cancer metastasis-associated microRNAs (miRNAs) have not been completely examined. Using a miRNA array, we previously determined that a significantly decreased level of miR-516a-3p was closely associated with the peritoneal dissemination of scirrhous gastric cancers. We previously established HSC-58 cells from a scirrhous gastric cancer patient, and we further isolated metastatic cells (58As9) from ascites in nude mice upon repeated orthotopic transplantation of HSC-58. In our previous report (Takei Y, et al. Cancer Res, 2011), we showed that the miR-516a-3p expression was significantly low and the peritoneal dissemination was significantly high in 58As9 compared with HSC-58. To augment the decreased expression of miR-516a-3p in 58As9 cells, we successfully prepared a cell line (58As9-miR-516a-3p) that stably overexpressed the miRNA. In the present study, we showed that orthotopic transplantation of 58As9-miR-516a-3p into nude mice resulted in significantly decreased primary tumor growth, ascites, and peritoneal dissemination. Moreover, the transplanted nude mice showed long survival time, probably due to the small amount of ascitic fluids pooled. The miRNA directly targeted sulfatase 1, which works to remove a sulfate group from heparan sulfate proteoglycans on the cell surface, and promotes the release of membrane-bound Wnt ligands into medium. Significantly increased concentrations of Wnt3a, Wnt5a, and nuclear-accumulated $\beta$-catenin were observed in 58As9 cells, and in 58As9-miR-516a-3p, all of the levels were attenuated. Anti-metastatic therapy via injection of the miR-516a-3p expression vector/atelocollagen mixture into 58As9 orthotopic tumors in nude mice resulted in significantly prolonged survival along with the inhibition of ascites and peritoneal dissemination. Our findings thus indicate that the miR-516a-3p-sulfatase $1-\mathrm{Wnt} \beta$-catenin route can be targeted to block the peritoneal dissemination of scirrhous gastric cancers.
\end{abstract}


Medical Research Archives, Vol. 5, Issue 7, July 2017

The microRNA miR-516a-3p regulates the Wnt pathway by targeting extracellular sulfatase 1 in human scirrhous gastric cancers: Anti-metastatic therapy via miRNA-based medicine

\section{Introduction}

Gastric cancer is the second leading cause of cancer-associated death worldwide $[1,2]$. Although surgical treatment is effective for gastric cancers in the early stage, in the advanced stages total gastrectomy and extended radical gastrectomy are often required but lead to poor prognosis due to post-surgical recurrence in the form of lymphatic spread, blood-borne metastasis, or peritoneal dissemination [1]. Scirrhous gastric carcinoma, a diffusely infiltrating type of poorly differentiated gastric carcinoma, is characterized by rapid cancer cell infiltration and proliferation accompanied by extensive stromal fibrosis [3]. Scirrhous gastric carcinoma accounts for approximately $10 \%$ of gastric carcinomas, and shows an extremely poor 5-year survival rate, compared with that of other types of gastric carcinomas [4]. Common features of scirrhous gastric cancer include progressive invasion and frequent metastasis to the peritoneum [5]. These points differ from intestinal types of gastric cancer [6]. Although attempts have been made to use chemotherapy, hormonal therapy, hyperthermia and immunotherapy to prevent peritoneal dissemination from scirrhous gastric carcinoma, these therapies were not effective [7]. There is thus a longstanding need for novel therapies based on the biological characteristic of scirrhous gastric cancer.

MicroRNAs (miRNAs) have received significant attention as a new class of non-coding RNAs engaged in the regulation of gene expression $[8,9]$. miRNAs are short 18-25 nucleotide stretches of RNA that bind to the target mRNAs and suppress their translation or initiate decay [9]. miRNAs exhibit differential expression in cancers and play important roles in their progression and metastasis [10, 11]. Recently, we identified various miRNAs [12] associated with peritoneal dissemination using our orthotopic inoculation models [13, 14]. In subsequent studies, we used our two established parent cell lines (HSC-44PE and HSC-58) from individual scirrhous gastric cancer patients [13], and further isolated two clones (44As3 and 58As9) with a highly metastatic character [14] from the corresponding parental cells. The results of an miRNA array among the above four lines showed that the miR-516a-3p levels were significantly lower in both of the metastatic lines than in their parent lines [12]. A stable line of 44As3 cells exhibiting ectopic overexpression of the miRNA miR-516a-3p (44As3-miR-516a-3p) exhibited decreased cell proliferation, migration, and invasion in vitro, and also decreased ascites fluid and peritoneal dissemination upon orthotopic inoculation into nude mice, when compared with an empty vector-inoculated group [12]. Further, 
Medical Research Archives, Vol. 5, Issue 7, July 2017

The microRNA miR-516a-3p regulates the Wnt pathway by targeting extracellular sulfatase 1 in human scirrhous gastric cancers: Anti-metastatic therapy via miRNA-based medicine

we showed that miR-516a-3p directly targets extracellular sulfatase 1 (SULF1), which is known to remove a sulfate group from heparan sulfate proteoglycans on the cell surface $[15,16]$, causing release of membrane-bound Wnt ligands from cells. Thus, in 44As3 cells, we have shown that miR-516a-3p regulates the Wnt pathway via SULF1 [12].

Here, we used 58As9 cells to prepare another cell line (58As9-miR-516a-3p) with stable ectopic overexpression of miR-516a-3p, and investigated its phenotypes both in vitro and in vivo. The finding that both metastatic lines exhibited a common phenotype of stable overexpression of miR-516a-3p provides strong evidence that the miR-516a-3p-SULF1-Wnt pathway is critical to the peritoneal dissemination of scirrhous gastric cancers, and therefore a suitable target for the inhibition of this dissemination. Moreover, we describe a method for reinforcing the desired miRNA overexpression in orthotopically inoculated tumors using an atelocollagen-mediated miRNA expression vector-delivery method. This method is useful for constitutive upregulation of miRNA levels in tumors via an artificial vector.

\section{Materials and Methods}

\subsection{Cancer cells}

HSC-58 cells were previously established from a scirrhous gastric cancer patient [13]. Orthotopic transplantation of HSC-58 cells into BALB/c nude mice (male, 6-weeks-old: Japan SLC, Hamamatsu, Japan) resulted in peritoneal dissemination (including to the omentum or mesenterium), and ascites in a small number of mice. Twelve cycles of i) isolation and ii) orthotopic transplantation of the tumor cells from ascites were vigorously repeated. In this way, a metastatic cell line (58As9) with a strong capacity for inducing ascites and strong peritoneal dissemination properties was successfully isolated [14]. All of the cells were cultured in RPMI 1640 supplemented with 10\% FBS [13, 14].

\subsection{RNA isolation}

Total RNA was extracted from the cells or tumor tissues using TRIzol Reagent (Invitrogen), and the quality of the total RNA was evaluated by an Agilent 2100 Bioanalyzer (Agilent). The total RNA extracted was fractionated and cleaned up with a flashPAGE fractionator system (Ambion) to obtain pure miRNAs. 
The microRNA miR-516a-3p regulates the Wnt pathway by targeting extracellular sulfatase 1 in human scirrhous gastric cancers: Anti-metastatic therapy via miRNA-based medicine

\subsection{Quantitative real-time RT-PCR for miRNA (qRT-PCR)}

TaqMan ${ }^{\circledR}$ MicroRNA Assays (Applied Biosystems) were performed to quantitate the expression levels of mature miRNAs as described previously [12]. For the reverse-transcription step (10 ng total RNA per sample), a TaqMan ${ }^{\circledR}$ microRNA RT kit (Applied Biosystems) was used. All of the PCR reactions were run using a StepOne ${ }^{\mathrm{TM}}$ real-time PCR system (Applied Biosystems). The data on each row were processed by the $\Delta \mathrm{Ct}$ method [17].

\subsection{Stable ectopic overexpression of} miR-516a-3p in 58As9

The vector to express hsa-miR-516a-3p (1 $\mu \mathrm{g}$; Takara Bio, Shiga, Japan) was transfected into 58As9 cells using a Lipofectamine 2000 reagent as reported previously [12]. Details about this vector are given in Figure 1. The vector-transfected cells were selected with Geneticin ${ }^{\circledR}(100$ $\mu \mathrm{g} / \mathrm{ml}$ ), and a stable clone (termed 58As9-miR 516a-3p) that exhibited overexpression of miR-516a-3p was obtained.

\subsection{In vivo phenotypes of tumor} formation and peritoneal dissemination in 58As9-miR-516a-3p cells

The 58As9-miR-516a-3p cells $\left(1.5 \times 10^{6}\right.$ cells) were orthotopically transplanted into the middle wall of the greater curvature of the glandular portion of the stomach as described earlier [12]. Twenty-five days later, the primary tumors were removed and tumor wet weight was measured. Peritoneal dissemination to the omentum, mesenterim, and ascites was also examined. Animal experiments in the present study were performed in compliance with the guidelines of the Institute for Laboratory Animal Research at the National Cancer Center Research Institute or at Nagoya University Graduate School of Medicine.

\subsection{Luciferase reporter assay}

58As9-miR-516a-3p cells were seeded in six-well plates $\left(1.5 \times 10^{5}\right.$ per well $)$ and cultured for one day before transfection. The cells were then transfected with a pGL3 vector alone (Promega), the vector with SULF1-3'-UTR, or the vector with SULF1-3'-UTR but with mutation as reported previously [12]. A pRLTK vector $(0.1 \mu \mathrm{g}$; Promega) was co-transfected in order to normalize the transfection efficiency. Whole cell lysates with RIPA buffer (Thermo Scientific) were prepared $48 \mathrm{~h}$ after the transfection, and the Firefly and Renilla luciferase activities were assayed using a Dual-Luciferase Reporter assay kit (Promega) [12]. 
Medical Research Archives, Vol. 5, Issue 7, July 2017

The microRNA miR-516a-3p regulates the Wnt pathway by targeting extracellular sulfatase 1 in human scirrhous gastric cancers: Anti-metastatic therapy via miRNA-based medicine

\section{Figure 1}

Takei $Y$ et al

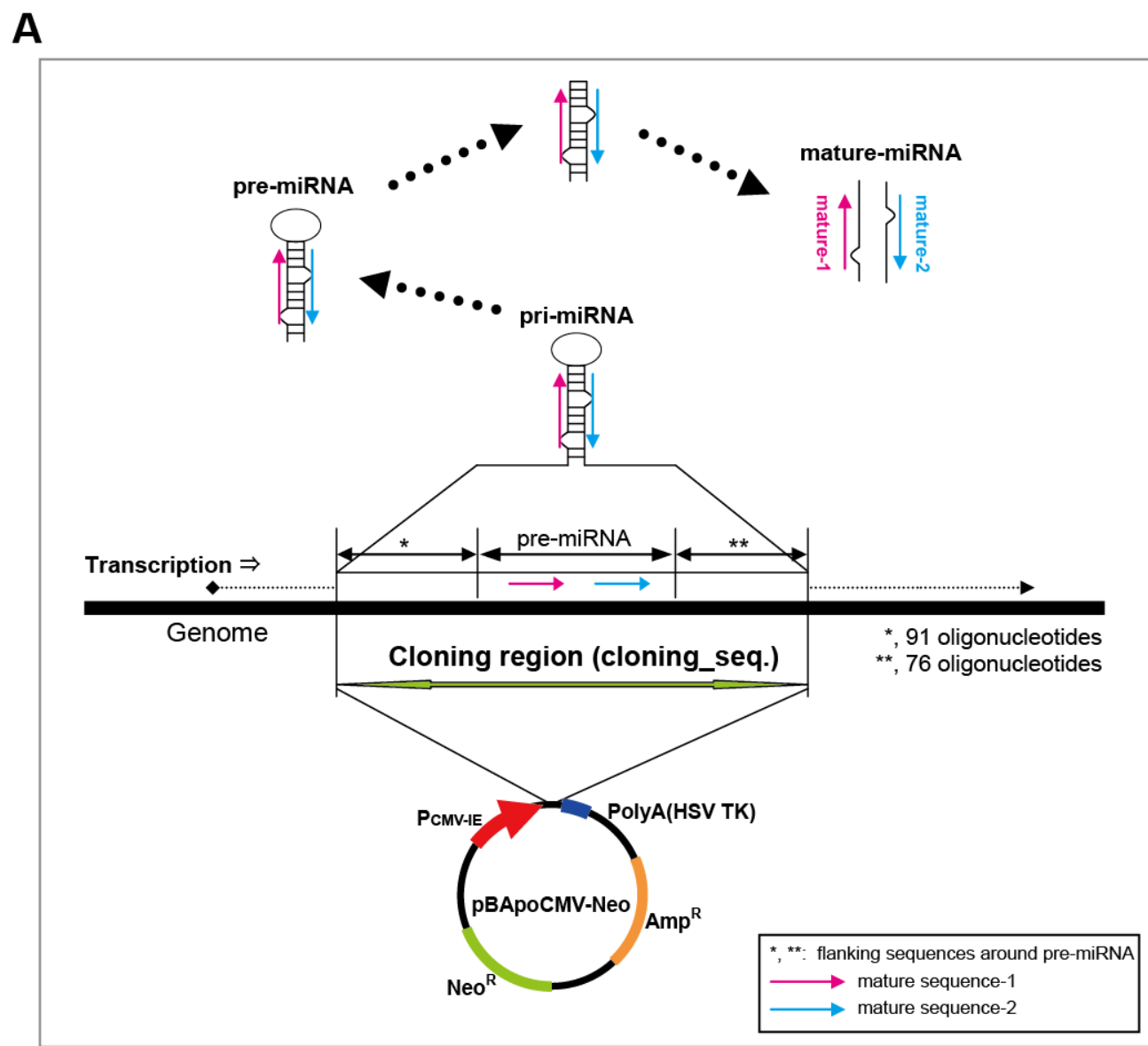

B

pre-miRNA sequence of hsa-mir-516a-1 (Stem-loop sequence: ID, MI0003180)

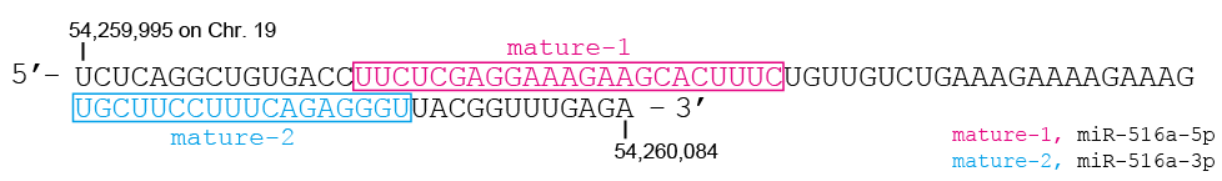

C

cloning sequence to transcribe the pri-miRnA
5'- TTAGTGAACCGGATCCGCATCTTTATGTTTCTGTCCATTTTAACTTGATCTAGGAAAATT
CCAACAAAAAACCCACGGTGCTGGAGCAAGATCTCAGGCTGTGACCTTCTCGAGGAAAGA
AGCACTTTCTGTTGTCTGAAAGAAAGAAAGTCTTCCTTCAGAGGGTACGGTTTGAG
AAAAGCAACGTTGAAGTTGACGCTGATCTTGGTAATACATTTGCAGAGCGTGCTGATCAT
CAAGCTTGGGGGAGGT - 3'

Figure 1. Information on the vector used for ectopic overexpression of miR-516a-3p. A, Schema of the vector design. B, Nucleotide sequences of pre-miRNA of mir-516a-1 (stem-loop sequence) containing mature-1 (miR-516a-5p) in red and mature-2 (miR-516a-3p) in blue. C, Cloning sequences used to transcribe the pri-miRNA with flanking sequences at the 5'-end (91 oligonucleotides) and 3'-end (76 oligonucleotides). The nucleotide sequences of pre-miRNA are shown in bold and underlined. 
The microRNA miR-516a-3p regulates the Wnt pathway by targeting extracellular sulfatase 1 in human scirrhous gastric cancers: Anti-metastatic therapy via miRNA-based medicine

\subsection{Isolation of nuclear proteins} from the cells

Nuclear and cytoplasmic proteins were separately isolated (ProteoJET ${ }^{\mathrm{TM}}$ Cytoplasmic and Nuclear Protein Extraction kit; Fermentas Life Sciences, York, UK) according to the manufacturer's protocol.

\subsection{Human Wnt3a and $\beta$-catenin}

\section{ELISA}

The amount of secreted Wnt3a or the amount of $\beta$-catenin was determined using an ELISA kit for human Wnt3a (MyBioSource, Inc., San Diego, CA), or DuoSet ${ }^{\circledR}$ IC human total $\beta$-catenin ELISA kit (R\&D Systems, Minneapolis, $\mathrm{MN}$ ), respectively.

\subsection{TCF/LEF reporter assay}

To monitor the activity of the Wnt signal-transduction pathway in the cells, a Cignal $^{\mathrm{TM}}$ TCF/LEF Reporter Assay kit was used (SABiosciences, Frederick, MD).

\subsection{Quantitative real-time RT-PCR for human SULF1}

Each total RNA (400 ng) isolated from the cells or the tumor tissues was reverse transcribed with a High Capacity cDNA Reverse Transcription Kit (Applied Biosystems). Quantitative PCR was performed using TaqMan Gene Expression Assays and a StepOne ${ }^{\mathrm{TM}}$ real-time PCR system (Applied Biosystems). Each row data was processed by the $\Delta \mathrm{Ct}$ method [17].
The expression level of human SULF1 (Assay ID, Hs00392839_m1) was normalized with hypoxanthine guanine phosphoribosyl transferase 1 (HPRT1; Applied Biosystems).

\subsection{Atelocollagen}

Atelocollagen is prepared from calf dermal collagen [24,25] and $0.5 \%$ atelocollagen is a liquid at $4^{\circ} \mathrm{C}$ and a gel at $37^{\circ} \mathrm{C}$. This biological feature contributes to the uptake of siRNA/miRNA or plasmid DNA vector upon direct injection into the tumors. Further, atelocollagen enhances the stability of siRNA/miRNA or plasmid DNA vector injected into tumors [19,23-25]. Atelocollagen is commercially available from Koken (Tokyo, Japan).

\subsection{Preparation of the miR-516a-3p expression vector/atelocollagen complex \\ To prepare the plasmid DNA vector/atelocollagen complex, equal volumes of atelocollagen in PBS and plasmid DNA solution were mixed by rotation for $20 \mathrm{~min}$ at $4^{\circ} \mathrm{C}[25]$.}

\subsection{Anti-metastasis therapy}

58As9 cells $\left(1.5 \times 10^{6}\right.$ cells $)$ were orthotopically inoculated into the stomach wall of 6-week-old BALB/c nude mice (Japan SLC) as described previously [14]. The cells were solved in $50 \mu$ of RPMI 1640 medium. Fifteen days later (day 0), intratumor (it) injection of the miR-516a-3p expression vector/atelocollagen mixture (total volume, $20 \mu \mathrm{l}$ ) was performed under 
The microRNA miR-516a-3p regulates the Wnt pathway by targeting extracellular sulfatase 1 in human scirrhous gastric cancers: Anti-metastatic therapy via miRNA-based medicine

anesthesia. An empty vector/atelocollagen mixture was also injected as a control. The total vector amount $(12.5 \mu \mathrm{g}, 25 \mu \mathrm{g}$, and 50 $\mu \mathrm{g})$ per tumor was examined. The final concentration of atelocollagen was $0.5 \%$. Five days later (on day 5), the injections were performed again. On day 15 , we sacrificed the mice and examined the excised primary tumor. The total RNA was isolated for qRT-PCR. For the controls, the HSC-58 parental cells were orthotopically inoculated, and the inoculated mice were observed without any therapy. On day 15 their tumors were excised. The survival rate was determined throughout the therapy $(n=20$ mice in each group).

\subsection{Statistical analysis}

The data were analyzed using the Mann-Whitney $U$ test. Probability values of less than 0.05 were considered to indicate significant differences.

\section{Results}

miR-516a-3p expression vector. In our previous study [12], we showed that the miR-516a-3p level was significantly reduced in 58As9 compared with HSC-58 cells. Accordingly, we used 58As9 cells to prepare a line that stably and ectopically overexpressed this miRNA, and designated it 58As9-miR-516a-3p [12]. Our vector system for overexpression of miR-516a-3p is shown in Figure 1, along with the detailed nucleotide sequences of miR-516a-3p.

In vivo phenotypes of
58As9-miR-516a-3p
Quantitative RT-PCR revealed the successful overexpression of the miRNA miR-516a-3p in 58As9 cells [12]. In an in vitro study, 58As9-miR-516a-3p cells showed a significant decrease in cell proliferation, migration, and invasion compared with 58As9 cells stably transfected with the empty vector [12]. In this article, to examine in vivo phenotypes, we orthotopically inoculated cells of the 58As9-miR-516a-3p line into the stomach wall of nude mice. Twenty-five days later, we observed a reduction in tumor formation in the mice (Figure 2A). Of note, among the 58As9-miR-516a-3p-inoculated mice, only 3 of the 11 mice that formed tumors harbored ascites (Figure 2A). We also observed a reduction in peritoneal metastasis to the omentum or mesenterium in the mice inoculated with 58As9-miR-516a-3p (Figure $2 A)$. The primary tumors derived from 58As9-miR-516a-3p were significantly smaller than those derived from 58As9, or from 58As9-empty vector $(\mathrm{p}<0.01$, Figure 2B). The mice that harbored tumors derived from 58As9-miR-516a-3p survived for a significantly longer period of time compared with those harboring tumors derived from 58As9 or from the 58As9-empty vector $(\mathrm{p}<0.01$, Figure 2C). 
Medical Research Archives, Vol. 5, Issue 7, July 2017

The microRNA miR-516a-3p regulates the Wnt pathway by targeting extracellular sulfatase 1 in human scirrhous gastric cancers: Anti-metastatic therapy via miRNA-based medicine

\section{Figure 2}

\section{Takei Y et al}

$\mathbf{A}$

\begin{tabular}{rcrrr}
\hline \multirow{2}{*}{ Types of cells } & $\begin{array}{c}\text { Tumor } \\
\text { formation }\end{array}$ & Ascites & \multicolumn{2}{c}{ Disseminated metastasis } \\
\cline { 4 - 5 } & & Omentum & Mesenterium \\
\hline HSC-58 & $10 / 15$ & $1 / 10$ & $1 / 10$ & $1 / 10$ \\
58As9 & $15 / 15$ & $15 / 15$ & $15 / 15$ & $14 / 15$ \\
58As-empty vector & $15 / 15$ & $14 / 15$ & $14 / 15$ & $13 / 15$ \\
58As9-miR-516a-3p & $12 / 15$ & $5 / 12$ & $5 / 12$ & $5 / 12$ \\
\hline
\end{tabular}

B

\section{Primary tumor growth}

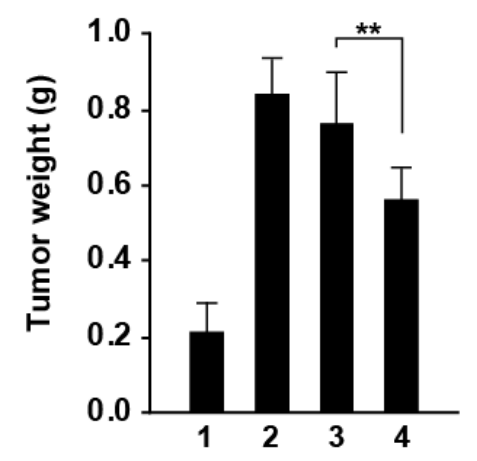

1: HSC-58 (tumors formed: $n=10$ )

2: 58 As 9 (tumors formed: $n=15$ )

3: 58As9-empty vector

(tumors formed: $n=15$ )

4: 58As9-miR-516a-3p

(tumors formed: $\mathrm{n}=12$ )
C

\section{Survival}

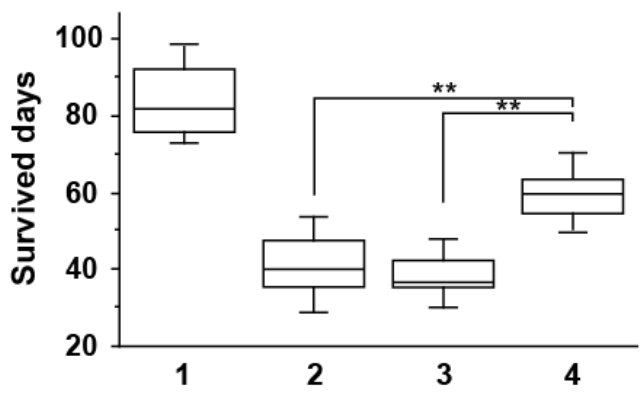

1: HSC-58

2: 58 As 9

3: 58As9-empty vector

4: 58As9-miR-516a-3p

Figure 2. In vivo phenotypes of tumor formation and peritoneal dissemination in 58As9-miR-516a-3p (stably miR-516a-3p-overexpressing 58As9 cells). A, Decreased tumor formation, ascites, and peritoneal dissemination to the omentum and mesenterium in the miRNA-overexpressing cells. Each cell was orthotopically inoculated into the nude mice, and 25 days later, all of the mice were sacrificed. In each group, 15 mice were used. B, Primary tumor growth. **, p<0.01. C, Survival period (days) of the nude mice. On day 0 , we also inoculated four kinds of cells into the stomach wall of nude mice $(\mathrm{n}=15$ nude mice in each group). ${ }^{* *}, \mathrm{p}<0.01$. 
Medical Research Archives, Vol. 5, Issue 7, July 2017

The microRNA miR-516a-3p regulates the Wnt pathway by targeting extracellular sulfatase 1 in human scirrhous gastric cancers: Anti-metastatic therapy via miRNA-based medicine

miR-516a-3p directly targets SULF1 (Figure 3A). Quantitative RT-PCR extracellular sulfatase 1 (SULF1). We revealed that SULF1 expression was showed by a luciferase reporter assay in significantly reduced in concert with 58As9 that the miRNA directly targeted overexpression of miR-516a-3p (Figure 3B).

\section{Figure 3}

\section{Takei $Y$ et al}
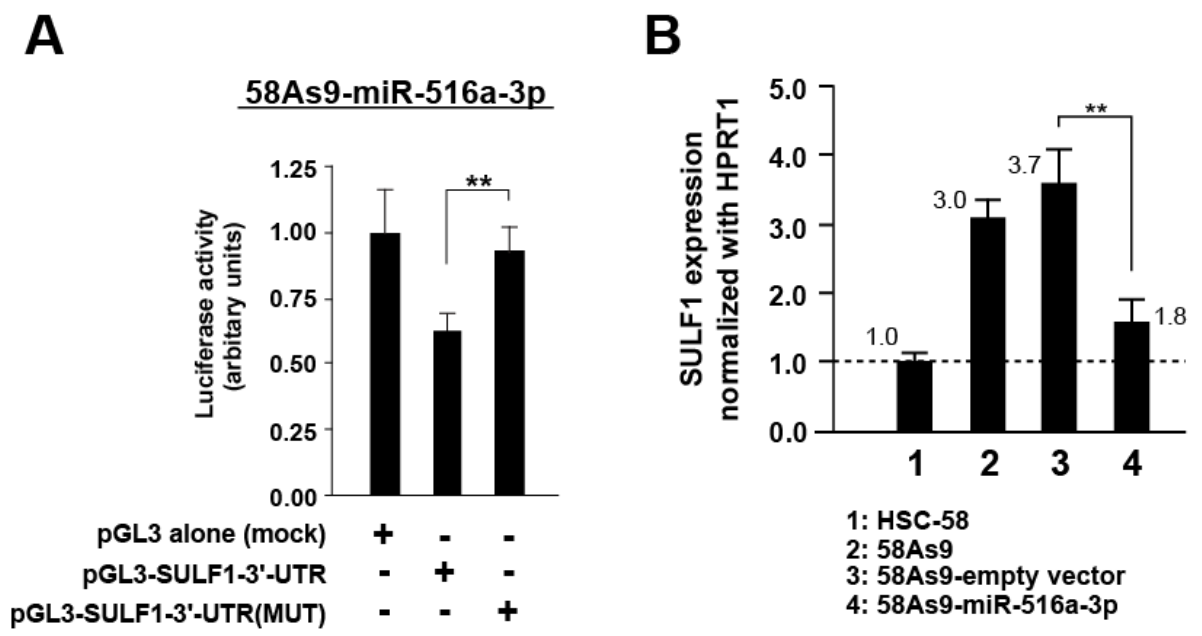

Figure 3. miR-516a-3p directly targets SULF1. A, Luciferase reporter assay. The SULF1 3'-UTR (bases from 2241-2273, 33 oligonucleotides) was inserted into pGL3 luciferase vector (Promega) as described earlier (Takei Y, et al: Cancer Res 2011). The mutated 3'-UTR was also inserted into the vector as a control. Each vector was transiently transfected into 58As9-miR-516a-3p cells. Finally, we performed a dual-luciferase assay. The bars show the means \pm SD $(n=4) .{ }^{* *}, p<0.01$. B, qRT-PCR analysis of SULF1. The SULF1 expression was normalized with HPRT1. The ratios of the expression level are shown in the graph (the level of HSC-58 was defined as 1.0). The bars show the means $\pm \mathrm{SD}(\mathrm{n}=4)$. **, $\mathrm{p}<0.01$. 
Medical Research Archives, Vol. 5, Issue 7, July 2017

The microRNA miR-516a-3p regulates the Wnt pathway by targeting extracellular sulfatase 1 in human scirrhous gastric cancers: Anti-metastatic therapy via miRNA-based medicine

miR-516a-3p regulates the Wnt pathway by targeting SULF1. SULF1

belongs to the extracellular sulfatases [15] and functions in internal glucosamine-6-sulfate $(6 \mathrm{~S})$ removal within heparan sulfate proteoglycans (HSPGs), thereby modulating the interactions of HSPGs with various signaling molecules [16, 26, 27]. Indeed, SULF1 promotes Wnt signaling [16, 26, 27]. An increased secretion of both Wnt3a and Wnt5a was observed in 58As9 cells (Figure 4A), and further, 58As9-miR-516a-3p cells showed a significant decrease in secretion of the Wnt ligands $(\mathrm{p}<0.05$, Figure $4 \mathrm{~A})$. The amount of $\beta$-catenin in the cytoplasmic and nuclear fractions of 58As9 was increased and the amount was significantly decreased in 58As9-miR-516a-3p cells $(\mathrm{p}<0.01$, Figure 4B). The TCF/LEF activity was high in 58As9 cells, but was significantly decreased in 58As9-miR-516a-3p cells compared to 58 As9-empty vector ( $\mathrm{p}<0.01$, Figure $4 \mathrm{C})$.

\section{miR-516a-3p vector-based anti-me-}

tastatic therapy. To examine the feasibility of using the miRNA-expression vector for anti-metastatic therapy, we injected the vector complexed with atelocollagen into 58As9 orthotopic tumors in nude mice. Atelocollagen was used as a delivery vehicle for the miRNA-expression vector into the cells $[12,25]$. The therapeutic procedures are shown in Figure 5A. When we injected the vector with atelocollagen into the 58As9 tumors, the expression levels of the miRNA were increased and the levels of SULF1 were decreased (Figure 5B). Both changes in expression levels were significant $(\mathrm{p}<0.01)$. Using our therapy protocol, the survival period of the treated mice became significantly longer than that of the control group mice (Figure 5C).

\section{Discussion}

Although tumor metastasis accounts for $90 \%$ of cancer-related deaths $[31,32]$, most existing cancer therapies were not developed to specifically block the metastasis process. Both pharmaceutical companies and academia must invest more in cancer metastasis research. This relative lack of resources committed to the study and understanding of metastasis is no doubt partly due to the subject's complexity. Therefore, to elucidate the mechanisms underlying cancer metastasis, we recently established a reliable model for peritoneal dissemination via orthotopic transplantation of human scirrhous gastric cancers $[13,14]$, and we have already shown the alteration of various gene expressions, including miRNAs, in this model [12]. 
Medical Research Archives, Vol. 5, Issue 7, July 2017

The microRNA miR-516a-3p regulates the Wnt pathway by targeting extracellular sulfatase 1 in human scirrhous gastric cancers: Anti-metastatic therapy via miRNA-based medicine

\section{Figure 4}

Takei Y et al

A

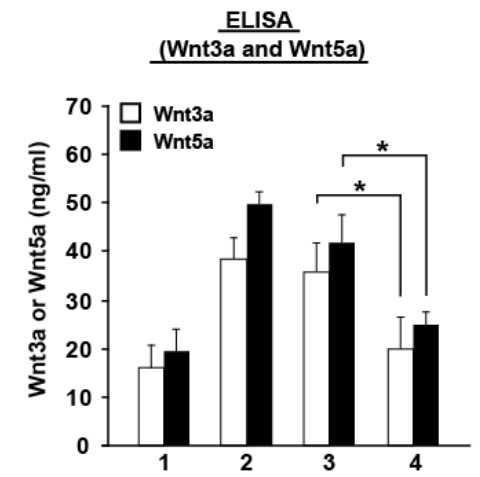

C

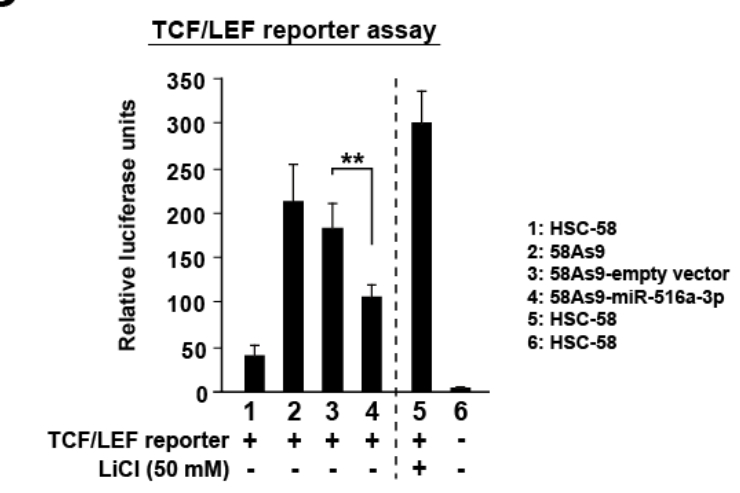

B

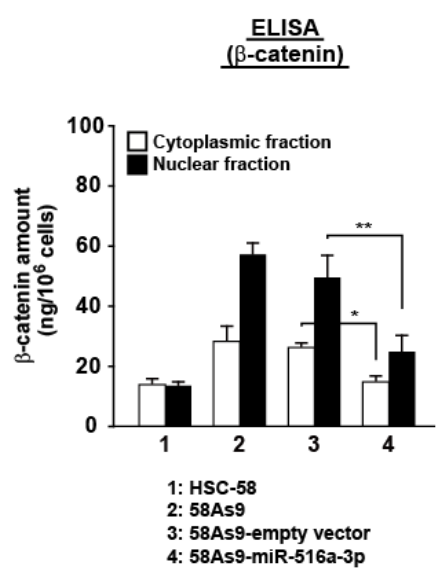

D

CD44 and Cyclin D1 Expression

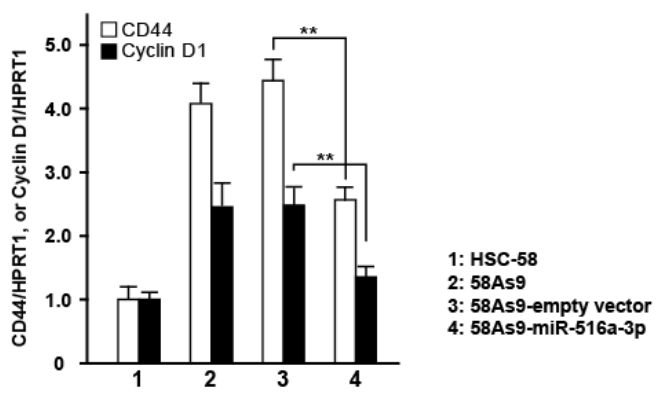

Figure 4. miR-516a-3p regulates the Wnt canonical pathway via SULF1 expression. A, ELISA of Wnt3a and Wnt5a. White bars, Wnt3a; black bars, Wnt5a. The bars show the means \pm SD $(n=4) . *, p<0.05$. B, ELISA measurement of $\beta$-catenin in both the cytoplasmic and nuclear fraction. The bars show the means \pm SD $(n=4)$. *, $\mathrm{p}<0.05$; and **, $\mathrm{p}<0.01$. C, TCF/LEF reporter assay. $\mathrm{LiCl}(50 \mathrm{mM})$, a GSK inhibitor was added to the cultures of HSC-58. This experiment served as a positive control to confirm that the assay system is was functional. Firefly luciferase activity was normalized with the Renilla luciferase activity. The bars show the means \pm SD $(n=4)$. ${ }^{* *}, \mathrm{p}<0.01$. D, qRT-PCR analysis of CD44 and cyclin D1. Their expressions were normalized with HPRT1. The bars show the means \pm SD $(n=4)$. **, $p<0.01$. White bars, CD44; black bars, cyclin D1. 
Medical Research Archives, Vol. 5, Issue 7, July 2017

The microRNA miR-516a-3p regulates the Wnt pathway by targeting extracellular sulfatase 1 in human scirrhous gastric cancers: Anti-metastatic therapy via miRNA-based medicine

\section{Figure 5}

Takei Y et al
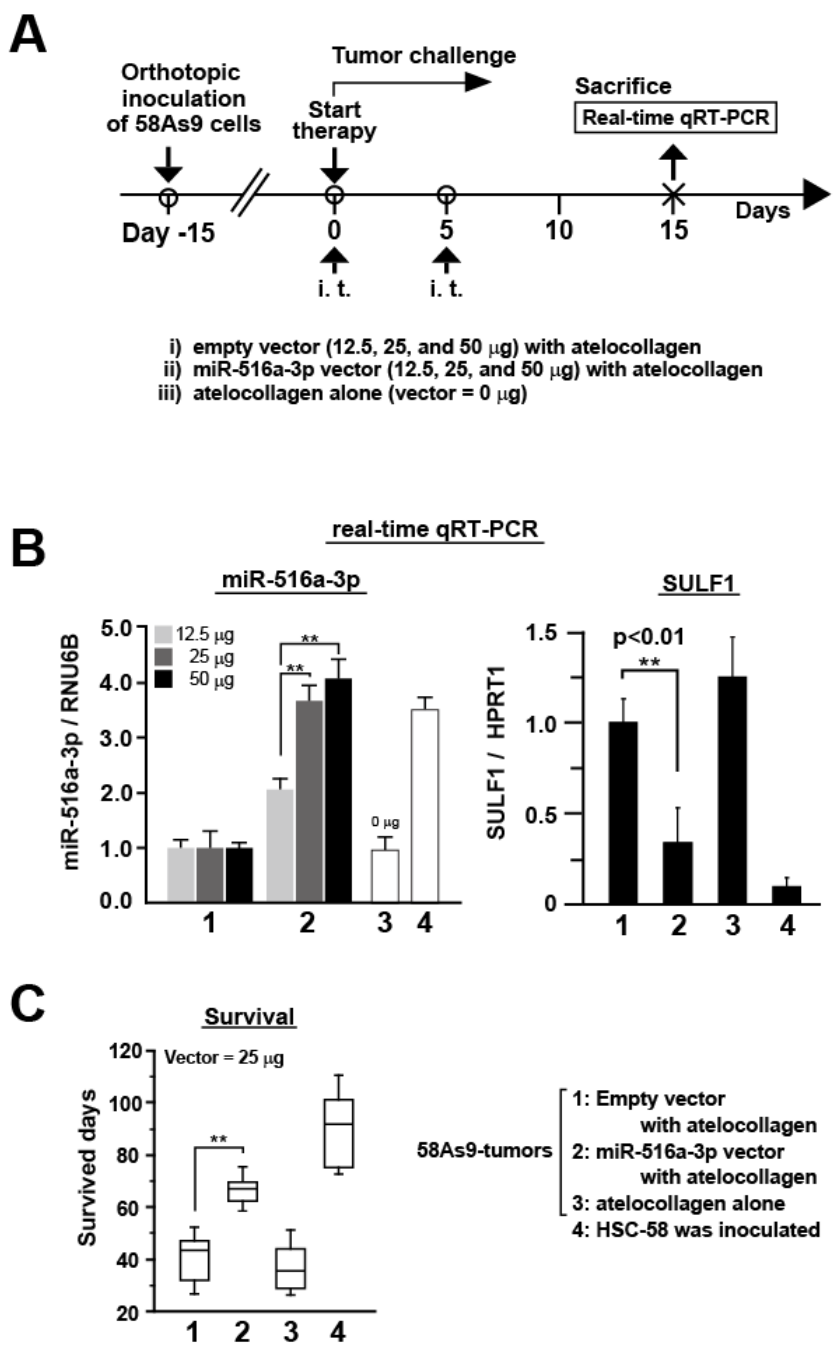

Figure 5. Inhibition of the miR-516a-3p expression vector upon peritoneal dissemination of 58As9. A, Experimental procedure. First, 58As9 cells were orthotopically inoculated into the stomach wall of nude mice. Fifteen days later, nude mice with 58As9 tumors were intratumorally injected with an empty vector or the miR-516a-3p vector complexed with atelocollagen (day 0). Three doses of the vector $(12.5,25$, and $50 \mu \mathrm{g} /$ tumor) were examined. On day 5, the same injection was performed again, and on day 15, all the mice were sacrificed, and the excised tumors were examined for qRT-PCR. As a control, atelocollagen alone (i.e., the delivery vehicle) was injected. As another control, HSC-58 (parental cells) inoculation was also performed. B, qRT-PCR analysis of miR-516a-3p and SULF1. The bars show the means $\pm S D(n=6) . * *, p<0.01$; NS, not significant. C, Survival period (days) of the mice. The vector $(25 \mu \mathrm{g} /$ tumor $)$ was administered. The bars show the means $\pm \mathrm{SD}(\mathrm{n}=6)$. ${ }^{* *}, \mathrm{p}<0.01$. 
Medical Research Archives, Vol. 5, Issue 7, July 2017

The microRNA miR-516a-3p regulates the Wnt pathway by targeting extracellular sulfatase 1 in human scirrhous gastric cancers: Anti-metastatic therapy via miRNA-based medicine

Recently, a specialized family of miRNAs, which Hurst et al. named metastamir [10], has been shown to have proand antimetastatic effects [11, 33]. In a previous report [12], we identified many kinds of metastamir that were potently associated with peritoneal disseminations of scirrhous gastric carcinomas. Here, our peritoneal dissemination model revealed a significantly decreased expression of miR-516a-3p in a highly metastatic cell line, 58As9, compared with the parental line, HSC-58. Orthotopic transplantation of 58As9-miR-516a-3p (an ectopic cell line with stable overexpression the miRNA) into nude mice resulted in a significantly longer survival time when compared with transplantation of 58As9-empty vector control cells. These phenotypes actually suggested that miR-516a-3p exhibits an anti-metastatic function in scirrhous gastric cancers. Indeed, in normal stomach tissues the expression level of the miRNA was extremely high. In contrast, the expression level of miR-516a-3p in patients with gastric cancers was significantly lower than in controls, and the degree of decrease was dependent on the peritoneal dissemination [12].

miR-516a-3p directly targeted SULF1 in both the 58As9 (Figure 3) and 44As3 [12] cell lines, and these highly metastatic derivatives maintained a high amount of
SULF1 protein due to the lack of regulation by the miRNA. SULF1 removes 6-O-sulfates from heparan sulfate proteoglycans on the cell surface, and induces a vigorous release of membrane-bound Wnt3a and Wnt5a ligands from the cells (Figure 4). Finally, the canonical Wnt pathway was constitutively activated in our highly metastatic derivatives, and activation of this pathway was connected to the increased expression levels of both CD44 and cyclin D1, which are specific target genes of the TCF/LEF family (Figure 4). It is of note that the ectopic enforced overexpression of the miR-516a-3p in both 44As3 and 58As9 showed similar phenotypes, such as significant suppression of cell proliferation, migration, and invasion in vitro. Further, when orthotopically inoculated into nude mice, these two miR-516a-3poverexpressing cell lines elicited significant inhibition of primary tumor growth, and prolonged survival rates in vivo. The common phenotypes in the two miRNA-overexpressing lines provide clear evidence that the miR-516a-3p-SULF1-Wnt pathway is generally critical for the peritoneal dissemination of scirrhous gastric cancers, and therefore would be a suitable target for inhibiting this dissemination. Moreover, we demonstrated the feasibility of using the miRNA expression vector as a therapeutic agent against peritoneal dissemination via the atelocollagen-mediated vector delivery method [25, 34]. Page | 14 
Medical Research Archives, Vol. 5, Issue 7, July 2017

The microRNA miR-516a-3p regulates the Wnt pathway by targeting extracellular sulfatase 1 in human scirrhous gastric cancers: Anti-metastatic therapy via miRNA-based medicine

Atelocollagen was used as a reagent to deliver the plasmid DNA into tumors [12].

We observed increased expression of CD44 and cyclin D1 as a result of TCF/LEF activation via $\beta$-catenin accumulated in the nucleus in the highly metastatic cell lines. The increase of cyclin D1 explicitly demonstrates the cell proliferation advantages of the highly metastatic cell lines compared with the corresponding parental lines. We have previously shown that these two metastatic lines proliferate more rapidly than the corresponding parental lines [14]. CD44, which is a major adhesion protein on the extracellular matrix, is involved with not only physiological processes including leukocyte homing and activation, and wound healing, but also tumor cell invasion and metastasis [28, 35, 36]. Now, it is clear that elevated CD44 is closely associated with cancer metastasis [28, 35, 36]. Thus, the highly metastatic cell lines acquire a suitable microenvironment for metastasis and dissemination of cancer cells, via elevated expression of the $\beta$-catenin target gene CD44. CD44 is also known as a marker of tumor-initiating cells (TICs) in various cancers, including gastric cancers [37]. TICs have been defined as a selected population of tumor cells that grow on serial transplantation in xenogeneic models [35]. TICs, like stem cells, are highly resistant to apoptosis and are thought to be essential for metastasis [35]. Indeed, in our experiments the highly metastatic lines revealed a high tumor formation ratio in nude mice upon orthotopic inoculation, and thus the elevation in CD44 caused by activation of the Wnt- $\beta$-catenin pathway has the potential to confer the characteristic of "stemness" observed in TICs to the highly metastatic lines. In any case, additional investigations using our models will be necessary to elucidate the mechanism connecting peritoneal dissemination and TICs.

In conclusion, we showed that miR-516a-3p regulates the canonical Wnt pathway by directly targeting SULF1, which is an enzyme that liberates ECM-bound Wnt ligands into the medium in human scirrhous gastric cancers. Further, we demonstrated that miR-516a-3p can be targeted in anti-metastatic therapy for inhibiting the peritoneal dissemination of gastric cancers. 
Medical Research Archives, Vol. 5, Issue 7, July 2017

The microRNA miR-516a-3p regulates the Wnt pathway by targeting extracellular sulfatase 1 in human scirrhous gastric cancers: Anti-metastatic therapy via miRNA-based medicine

\section{References}

1. Anderson C, Nijagal A, Kim J. Molecular markers for gastric adenocarcinoma: an update. Mol Diagn Ther 2006;10:345-52.

2. Takahashi I, Matsusaka T, Onohara T, Nishizaki T, Ishikawa T, Tashiro H, Wakasugi K, Kume K, Maehara Y, Sugimachi K. Clinicopathological features of long-term survivors of scirrhous gastric cancer. Hepatogastroenterology 2000;47:1485-8.

3. Nakazawa K, Yashiro M, Hirakawa K. Keratinocyte growth factor produced by gastric fibroblasts specifically stimulates proliferation of cancer cells from scirrhous gastric carcinoma. Cancer Res 2003;63:8848-52.

4. Liu Y, Yoshimura K, Yamaguchi N, Shinmura K, Yokota J, Katai H. Causation of Borrmann type 4 gastric cancer: heritable factors or environmental factors? Gastric Cancer 2003;6:17-23.

5. Hippo Y, Yashiro M, Ishii M, Taniguchi H, Tsutsumi S, Hirakawa K, Kodama T, Aburatani H. Differential gene expression profiles of scirrhous gastric cancer cells with high metastatic potential to peritoneum or lymph nodes. Cancer Res 2001;61:889-95.

6. Jass JR, Sobin LH, Watanabe H. The
World Health Organization's histologic classification of gastrointestinal tumors. A commentary on the second edition. Cancer 1990;66:2162-7.

7. Samel S, Singal A, Becker H, Post S. Problems with intraoperative hyperthermic peritoneal chemotherapy for advanced gastric cancer. Eur J Surg Oncol 2000;26:222-6.

8. Calin GA, Croce CM. MicroRNA signatures in human cancers. Nat Rev Cancer 2006;6:857-66.

9. Bartel DP. MicroRNAs: genomics, biogenesis, mechanism, and function. Cell 2004;116:281-97.

10. Hurst DR, Edmonds MD, Welch DR. Metastamir: the field of metastasis-regulatory microRNA is spreading. Cancer Res 2009;69:7495-8.

11. Ma L, Teruya-Feldstein J, Weinberg RA. Tumour invasion and metastasis initiated by microRNA-10b in breast cancer. Nature 2007;449:682-8.

12. Takei Y, Takigahira M, Mihara K, Tarumi Y, Yanagihara K. The metastasis-associated microRNA miR-516a-3p is a novel therapeutic target for inhibiting peritoneal dissemination of human scirrhous gastric 
The microRNA miR-516a-3p regulates the Wnt pathway by targeting extracellular sulfatase 1 in human scirrhous gastric cancers: Anti-metastatic therapy via miRNA-based medicine

cancer. Cancer Res 2011;71:1442-53.

13. Yanagihara K, Tanaka H, Takigahira M, Ino Y, Yamaguchi Y, Toge T, Sugano K, Hirohashi S. Establishment of two cell lines from human gastric scirrhous carcinoma that possess the potential to metastasize spontaneously in nude mice. Cancer Sci 2004;95:575-82.

14. Yanagihara K, Takigahira M, Tanaka H, Komatsu T, Fukumoto H, Koizumi F, Nishio $\mathrm{K}$, Ochiya T, Ino $\mathrm{Y}$, Hirohashi S. Development and biological analysis of peritoneal metastasis mouse models for human scirrhous stomach cancer. Cancer Sci 2005;96:323-32.

15. Morimoto-Tomita M, Uchimura K, Werb Z, Hemmerich S, Rosen SD. Cloning and characterization of two extracellular heparin-degrading endosulfatases in mice and humans. J Biol Chem 2002;277:49175-85.

16. Ai X, Do AT, Lozynska O, Kusche-Gullberg M, Lindahl U, Emerson CP Jr. QSulf1 remodels the 6-O sulfation states of cell surface heparan sulfate proteoglycans to promote Wnt signaling. J Cell Biol 2003;162:341-51.

17. Reinhart BJ, Slack FJ, Basson M, Pasquinelli AE, Bettinger JC, Rougvie AE, Horvitz HR, Ruvkun G. The 21-nucleotide let-7 RNA regulates developmental timing in Caenorhabditis elegans. Nature 2000;403:901-6.

18. Mu P, Nagahara S, Makita N, Tarumi Y, Kadomatsu K, Takei Y. Systemic delivery of siRNA specific to tumor mediated by atelocollagen: Combined therapy using siRNA targeting Bcl-xL and cisplatin against prostate cancer. Int J Cancer 2009;125:2978-90.

19. Takei Y, Kadomatsu K, Yuzawa Y, Matsuo S, Muramatsu T. A small interfering RNA targeting vascular endothelial growth factor as cancer therapeutics. Cancer Res 2004;64:3365-70.

20. Sherf BA., Navarro SL, Hannah RR, Waod KV. Dual-Luciferase ${ }^{\circledR}$ reporter assay: an advanced coreporter technology integrating firefly and Renilla luciferase assays. Promega Notes 1996;57:2-9.

21. Takei Y, Kadomatsu K, Matsuo S, Itoh H, Nakazawa K, Kubota S, Muramatsu $T$. Antisense oligodeoxynucleotide targeted to Midkine, a heparin-binding growth factor, suppresses tumorigenicity of mouse rectal carcinoma cells. Cancer Res 2001;61:8486-91.

22. Takei Y, Kadomatsu K, Itoh H, Sato W, Nakazawa K, Kubota S, Muramatsu T. 5'-, 
Medical Research Archives, Vol. 5, Issue 7, July 2017

The microRNA miR-516a-3p regulates the Wnt pathway by targeting extracellular sulfatase 1 in human scirrhous gastric cancers: Anti-metastatic therapy via miRNA-based medicine

3'-inverted thymidine-modified antisense oligodeoxynucleotide targeting midkine. Its design and application for cancer therapy. $\mathbf{J}$ Biol Chem 2002;277:23800-6.

23. Takei Y, Kadomatsu K, Goto T, Muramatsu T. Combinational antitumor effect of siRNA against midkine and paclitaxel on growth of human prostate cancer xenografts. Cancer 2006;107:864-73.

24. Takei Y, Kadomatsu K. In vivo delivery technique of nucleic acid compounds using atelocollagen: Its use in cancer therapeutics targeted at the heparin-binding growth factor midkine. Gene Ther Mol Biol;9:257-64.

25. Ochiya T, Takahama Y, Nagahara S, Sumita Y, Hisada A, Itoh H, Nagai Y, Terada M. New delivery system for plasmid DNA in vivo using atelocollagen as a carrier material: the Minipellet. Nat Med 1999;5:707-10.

26. Bafico A, Liu G, Goldin L, Harris V, Aaronson SA. An autocrine mechanism for constitutive Wnt pathway activation in human cancer cells. Cancer Cell 2004;6:497-506.

27. Nawroth R, van Zante A, Cervantes S, McManus M, Hebrok M, Rosen SD. Extracellular sulfatases, elements of the Wnt signaling pathway, positively regulate growth and tumorigenicity of human pancreatic cancer cells. PLoS One 2007;2:e392.

28. Wielenga VJ, Smits R, Korinek V, Smit L, Kielman M, Fodde R, Clevers H, Pals ST. Expression of CD44 in Apc and Tcf mutant mice implies regulation by the WNT pathway. Am J Pathol. 1999;154:515-23.

29. Tetsu O, McCormick F. Beta-catenin regulates expression of cyclin D1 in colon carcinoma cells. Nature 1999;398:422-6.

30. Shtutman M, Zhurinsky J, Simcha I, Albanese C, D'Amico M, Pestell R, Ben-Ze'ev A. The cyclin D1 gene is a target of the beta-catenin/LEF-1 pathway. Proc Natl Acad Sci U S A. 1999;96:5522-7.

31. Sleeman J, Steeg PS. Cancer metastasis as a therapeutic target. Eur $\mathrm{J}$ Cancer 2010;46:1177-80.

32. Steeg PS, Theodorescu D. Metastasis: a therapeutic target for cancer. Nat Clin Pract Oncol 2008;5:206-19.

33. Tavazoie SF, Alarcón C, Oskarsson T, Padua D, Wang Q, Bos PD, Gerald WL, Massagué J. Endogenous human microRNAs that suppress breast cancer metastasis. Nature 2008;451:147-52.

34. Honma K, Iwao-Koizumi K, Takeshita F, Yamamoto Y, Yoshida T, Nishio K, Nagahara S, Kato K, Ochiya T. RPN2 gene confers 
The microRNA miR-516a-3p regulates the Wnt pathway by targeting extracellular sulfatase 1 in human scirrhous gastric cancers: Anti-metastatic therapy via miRNA-based medicine

docetaxel resistance in breast cancer. Nat Med. 2008;14:939-48.

35. Zöller M. CD44: can a cancer-initiating cell profit from an abundantly expressed molecule? Nat Rev Cancer 2011;11:254-67.

36. Ishimoto T, Nagano O, Yae T, Tamada $M$, Motohara T, Oshima H, Oshima M, Ikeda T, Asaba R, Yagi H, Masuko T, Shimizu T, et al. CD44 Variant Regulates Redox Status in
Cancer Cells by Stabilizing the xCT Subunit of System xc(-) and Thereby Promotes Tumor Growth. Cancer Cell 2011;19:387-400.

37. Takaishi S, Okumura T, Tu S, Wang SS, Shibata W, Vigneshwaran R, Gordon SA, Shimada Y, Wang TC. Identification of gastric cancer stem cells using the cell surface marker CD44. Stem Cells 2009;27:1006-20.

\section{Acknowledgments}

Grant support: Grants-in-Aid from the Ministry of Education, Culture, Sports, Science, and Technology of Japan (17016030) and from the Japan Society for the Promotion of Science (17790185, 19590273, 21590305, and 24590348).
We thank Drs. Kazuo Kita and Takayuki Okubo for their helpful suggestions regarding the experiments. We also thank Ms. Tae Hayashi, and Misato Takigahira for their excellent technical assistance. 